\title{
A Runtime Performance Analysis for Web Service-Based Applications
}

\author{
Afef Mdhaffar, Soumaya Marzouk, Riadh Ben Halima, and Mohamed Jmaiel \\ University of Sfax, ReDCAD Laboratory, B.P. 1173, Sfax-Tunisia \\ \{afef.mdhaffar, soumaya.marzouk\}@redcad.org, \\ \{riadh. benhalima, mohamed.jmaiel\}@enis.rnu.tn
}

\begin{abstract}
Runtime performance evaluation is necessary for the continuous QoS (Quality of Service) management of Web service-based applications. The analysis is critical in service provisioning since it allows to detect QoS degradation and to identify its source. However, performance analysis in current applications is usually based on QoS reference values that are manually instrumented and pre-established independently from the execution context. It is inflexible to change. The paper extends our previous research on performance evaluation within a self-healing framework and proposes a novel analysis approach based on automatic generation of QoS reference values. These QoS reference values are generated whenever we start the application execution in a new context. This approach enables the detection of QoS degradation, the identification of its nature (like execution or communication level) and the localization of its cause (as scalability issue, node failure or network overload). The carried out experiments show that the dynamic generated QoS reference values are suitable and their associated analysis results are accurate.
\end{abstract}

Keywords: QoS reference values, Self-healing, Web services, Performance analysis.

\section{Introduction}

Web services become crucial and largely used in the design of distributed applications. To ensure their efficiency, Web services need to support self-healing properties in order to deal with dynamic execution context. To support such properties, a four phases process has been established [1. The first phase consists in monitoring the Web service and its hosting environment in order to prepare the necessary measures to the analysis phase. The latter uses these metrics to detect possible failures or QoS degradation, identify the degradation nature and localize its cause. In such case, the third phase takes place. It looks for an equivalent Web service to replace the degraded one. Then, the fourth phase enforces repair actions. In a previous work [2], we performed the analysis based on preestablished QoS reference values. Therefore, we experimented each Web service on a large scale environment [3] to determine its QoS reference values. This is

F. Daniel and F.M. Facca (Eds.): ICWE 2010 Workshops, LNCS 6385, pp. 313-324, 2010.

(C) Springer-Verlag Berlin Heidelberg 2010 
hard to do because it requires human competences, specific tools and time. In addition, these reference values are related to the execution context. So, we need to repeat experiments whenever we (i) update the service implementation, (ii) change the hosting server, and (iii) modify the execution context.

Many existing works deal with the performance analysis of Web services. We notice that all studied works 4,567789. rely on the use of a pre-established QoS reference values describing the expected performance of a Web service on a specific context. Such approaches are not suitable for dynamic context. In fact, it is very hard to manually generate QoS reference values for each Web service on every context. Also, we picked out that the majority of existing works 4 5 [6 6789 does not allow an accurate localization of the QoS degradation cause. It can lead to useless reconfiguration actions.

In this paper, we propose a novel approach of performance analysis based on automatic and dynamic generation of QoS reference values at runtime. This approach consists in (1) monitoring the variation of (i) QoS parameters values, (ii) node load and (iii) network load during a training period while using a previously developed QoS monitoring approach and specific tools to check the overload and (2) studying the variation of monitored values and the correlation between them. Through this study, we distinguish different reasoning scenarios in order to characterize the Web state and generate the QoS reference values from scratch. We implemented these scenarios as algorithms in order to automate the generation process. For instance, if the Web service state is acceptable1, the average of the monitored values represents the QoS reference values. Otherwise, the algorithm alerts about the performance degradation, characterizes its cause and enforces recovery actions. Our approach regenerates the QoS reference values whenever the Web service changes its execution context.

To illustrate the proposed approach, we carried out experiments on the Conference Management System (CMS). It is a real WS-based application that we developed in order to review management in scientific conferences and applicable for industrial review processes. First, we compare the QoS reference values that we generated automatically to a manually generated QoS reference values. The evaluation shows that both values are so close and the difference between them is negligible. So, we conclude that the automatically generated QoS reference values are reasonable. Second, we demonstrate that the performance analysis based on the automatically generated QoS reference values is achieved rigorously. It detects the performance degradation of a Web service, characterizes its nature, and locates its cause. It can specify whether the degradation has been occurred due to a scalability issue, or due to the network performance degradation or due to the node performance degradation.

This paper is organized as follows. Section 2 describes our contribution and details the generation process of the QoS reference values. Section 3 illustrates our approach. Section 4 discusses related work. Last section concludes the paper.

${ }^{1}$ A Web service execution/communication state is acceptable, if no execution/communication time degradation is detected. 


\section{A Performance Analysis Approach Based on Automatic and Dynamic Generation of QoS Reference Values}

We propose in this work a performance analysis approach for Web services. This approach enhances our previous QoS-Oriented Self-Healing middleware (QOSH) [2] while proposing a new analysis component. In our previous work [2], we used a pre-established QoS reference values for runtime analysis. This makes the used analysis approach [2] inflexible for many reasons. First, its application requires the elaboration of a large number of experiments to generate QoS reference values for each Web service. Second, we have to repeat experiments whenever the execution context changes. Also, our previous approach [2] does not allow an accurate localization of the degradation cause. So, we focus in this paper on (1) the automation of the QoS reference values generation for Web services and (2) the indentification of the degradation cause. Thus, QoS (execution and communication time) reference values are generated and updated when the execution context changes without human intervention. Then, our new analysis component uses these values to detect degradation, and it accurately identifies its nature and its cause. This approach is detailed in the following.

\subsection{Overview of Our Performance Analysis Approach}

Our performance analysis approach shown in Fig. 1 starts with checking the Web service execution state while studying data monitored during the training

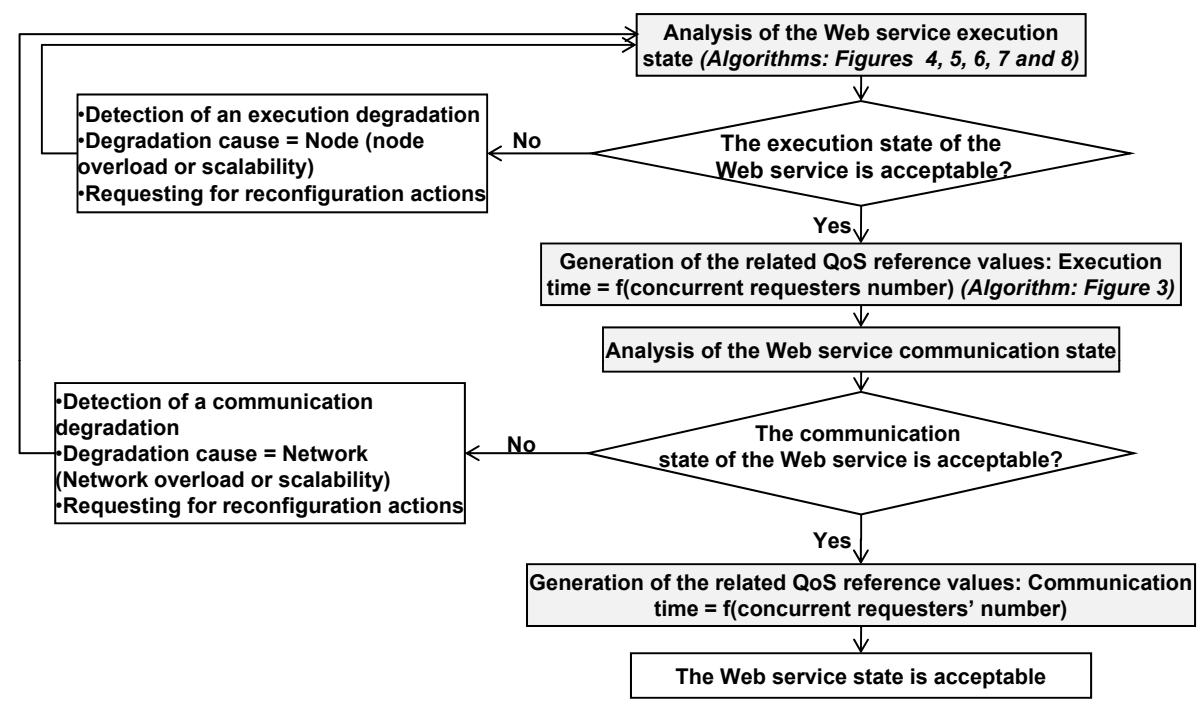

Fig. 1. Organigram of our performance analysis approach 
period 2. In case of an acceptable Web service execution state, our approach generates related QoS (execution time: Fig. 3) reference values and checks the Web service communication state. Then, it generates its related QoS (communication time) reference values in case of an acceptable communication state. Otherwise, when our approach detects a communication or execution degradation, it deduces its cause, requests for reconfiguration actions and restarts our analysis approach. In case of mobile Web services, our approach regenerates the QoS reference values if the concerned Web service migrates to another node. During execution, the established QoS reference values will be updated periodically for consolidation purposes. Our performance analysis approach detects QoS degradation, deduces its nature (execution or communication) and localizes its cause (scalability issue, node or network overload).

\subsection{A QoS Reference Values Generation Algorithm}

Fig. 22presents an algorithm that implements our performance analysis approach. It takes as inputs five parameters, which are collected during the monitoring phase. These parameters allow us to check the Web service state. They are detailed in Table 1 At the instant $\mathrm{t}_{i}$, we measure the execution time $\left(\operatorname{Texec}\left(t_{i}\right)\right)$, the request number $\left(\operatorname{NbReq}\left(t_{i}\right)\right)$ and the communication time $\left(\operatorname{Tcom}\left(t_{i}\right)\right)$, while

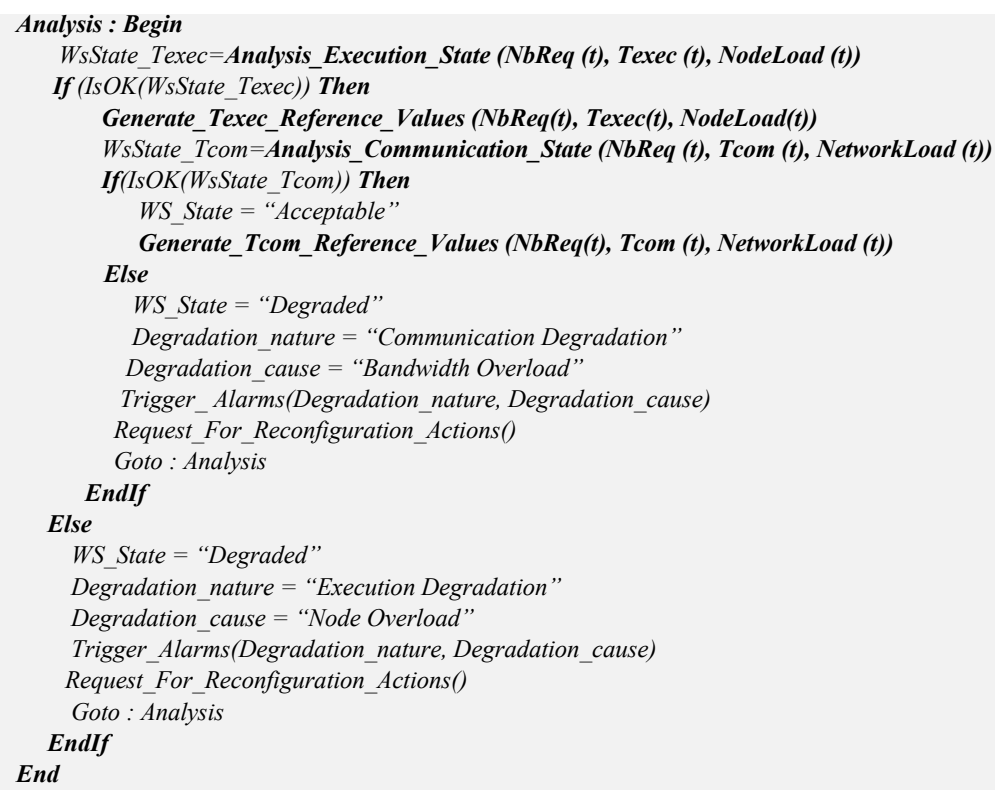

Fig. 2. Performance analysis algorithm

\footnotetext{
2 The training period is a time interval which gives us the possibility to assess the Web service performance using monitored QoS parameters, node and network load.
} 
Table 1. The inputs of the performance analysis algorithm

\begin{tabular}{ll}
\hline Inputs & Description \\
\hline NbReq(t) & $\begin{array}{l}\text { It presents the variation (Real) of the successive request number during the } \\
\text { training period T }\end{array}$ \\
Texec(t) & $\begin{array}{l}\text { It presents the variation (Real) of the execution time during the training } \\
\text { period } \mathrm{T}\end{array}$ \\
Tcom(t) & $\begin{array}{l}\text { It presents the variation (Real) of the communication time during the training } \\
\text { period T }\end{array}$ \\
NodeLoad(t) & It presents the variation (Real) of the node load during the training period T \\
NetworkLoad(t) & It presents the variation (Real) of the network load during the training period \\
\hline
\end{tabular}

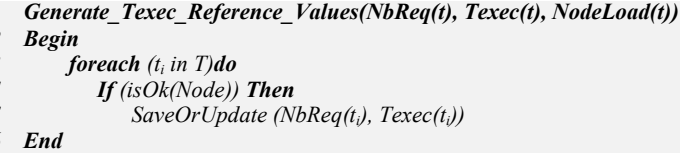

Fig. 3. The sub-algorithm: Generate_Texec_Reference_Values

adopting our monitoring approach [2]. To extract the network load value at instant $\mathrm{t}_{i}$ (NetworkLoad $\left.\left(t_{i}\right)\right)$, we used the IPERF 3 tool which performs the measurement of the network bandwidth. Finally, to measure the node load at instant $\mathrm{t}_{i}\left(\operatorname{NodeLoad}\left(t_{i}\right)\right)$, we have developed a specific checking_node_load service which evaluates the node load while checking the memory occupation variation and the response time variation of this node. The algorithm shown in Fig. 2 makes use of four sub-algorithms (emphasized in bold). The first one, Analysis_Execution_State (see Section 2.3), allows the execution time analysis of the Web service. The second one, Generate_Texec_Reference_Values (see Fig. 3), generates the execution time reference values. The third sub-algorithm, Analysis_Communication_State performs the analysis of the communication time. The fourth one, Generate_Tcom_Reference_Values, is very similar to the Generate_Texec_Reference_Values sub-algorithm and generates the communication time reference values. It consists on saving or updating (NbReq(ti), Tcom(ti)) when the network is not overloaded. The execution time analysis and the communication time analysis sub-algorithms follow the same way of reasoning. So, we detail only the execution time analysis sub-algorithm.

\subsection{Execution Time Analysis Sub-algorithm}

The main idea of the execution time analysis sub-algorithm (Analysis_Execution_ State (NbReq(t), Texec(t), NodeLoad $(t)))$ is to study the positive correlation between $N b R e q(t)$ and Texec $(t)$. In fact, these two distributions should be correlated to reflect a normal behavior of the Web service. The correlation study is based on the computing of the correlation coefficient value using the equation (1) [10].

3 http://iperf .sourceforge.net/ 


$$
\rho=\frac{\sum\left(x_{i}-\bar{x}\right) *\left(y_{i}-\bar{y}\right)}{\sqrt{\sum\left(x_{i}-\bar{x}\right)^{2}} * \sqrt{\sum\left(y_{i}-\bar{y}\right)^{2}}} \quad \text { with } \quad \bar{x}=\frac{1}{N} * \sum\left(x_{i}\right)
$$

If the correlation coefficient value $\rho$ is greater than 0.8 [10, we conclude that NbReq( $t$ ) [x] and Texec(t) [y] are positively correlated. Then, the execution time analysis sub-algorithm begins with checking the positive correlation between NbReq(t) and Texec(t). If they are positively correlated (Fig. 4. line 1), it concludes that the behavior of the concerned Web service is normal but it can't deduce that the execution state of this Web service is acceptable. Therefore, a continuous degradation of the concerned Web service performance can also lead to a positive correlation of these two distributions. As a result, we check the hosting node load to evaluate the state of the concerned Web service. If the hosting node is not overloaded (Fig. 4. line 2), this sub-algorithm concludes that the Web service execution state is acceptable (Fig. 4: line 3). Otherwise (Fig. 4. line 4), it deduces that the degradation comes from the execution time (Fig. 4 . lines 5 and 6 ) and is related to a scalability issue (Fig. 4. line 7). In fact, the hosting node can't support this important number of simultaneous requests.

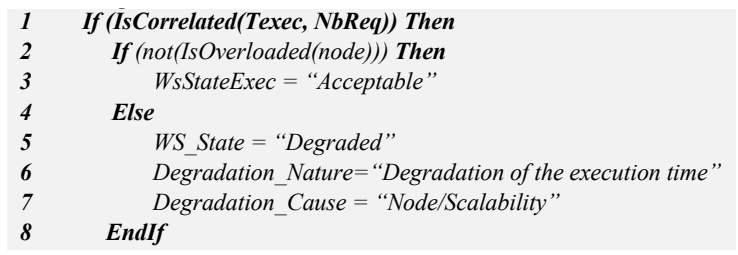

Fig. 4. Execution time analysis sub-algorithm - Part 1: The two distributions are positively correlated

If $N b R e q(t)$ and $T e x e c(t)$ are not positively correlated, the execution time analysis algorithm checks the variation of $\operatorname{Texec}(t)$ to examine the Web service state. It distinguishes between four main cases: (i) Texec(t) is increasing (Fig. 55), (ii) Texec(t) is decreasing (Fig. 6), (iii) Texec(t) is constant (Fig. 7) and (iv) Texec $(t)$ follows a random variation (Fig. 8).

We detail the case when Texec(t) is increasing and not positively correlated with $N b R e q(t)$ (Fig. 5). The non correlation between these two distributions implies that the increase of the execution time value is not related to the simultaneous request number variation. Thus, it is related to a node performance decrease. In this case, we have to know if this node performance decrease is critical in order to characterize the concerned Web service state. This is achieved by checking the hosting node load. If the node is not overloaded (Fig. 5. line 3), our algorithm deduces that the execution state of the concerned Web service is acceptable (Fig. 5] line 4). Otherwise (Fig. 5. line 5), we deduce that an execution degradation has occurred (Fig. 5. lines 6 and 7). To locate the degradation cause, we analyze $N b R e q(t)$. If it is constant or decreasing (Fig. 5 line 8), we conclude 


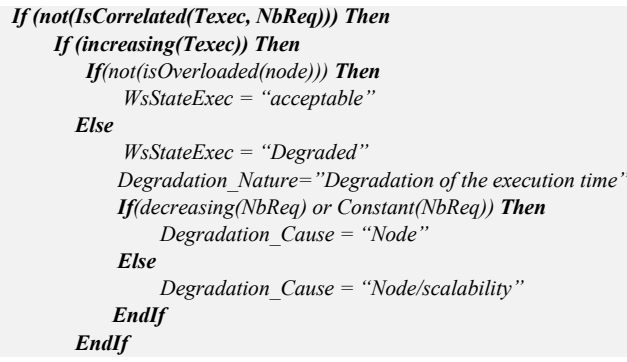

Fig. 5. Execution time analysis subalgorithm - Part 2.1: Increase of the execution time during the training period

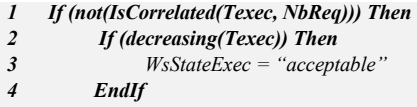

that the hosting node is the cause of the degradation (Fig. 5. line 9). Otherwise, (i.e. the variation is random) our algorithm deduces that the degradation would be related to a scalability issue or a node overload (Fig. 55, line 11).

The execution time analysis algorithm handles the case when Texec $(t)$ is decreasing and not correlated with $N b R e q(t)$ in Fig. 6, It deduces that the Web service state is acceptable. In fact, the decrease of the execution time value implies that the hosting node offers a better QoS.

The algorithm shown in Fig. 7 deals with the case when Texec(t) is constant and not correlated with $N b R e q(t)$. It analyzes the hosting node load to characterize the Web service state. If the hosting node is not overloaded (Fig. 7] line 3 ), it concludes that the Web service execution state is acceptable (Fig. 7) line 4). In the opposite case (Fig. 7) line 5), we deduce that an execution time degradation has occurred (Fig. 7) lines 6 and 7). To locate the degradation cause, this sub-algorithm analyzes the variation of $N b R e q(t)$. If it decreases (Fig. 7 line 8),

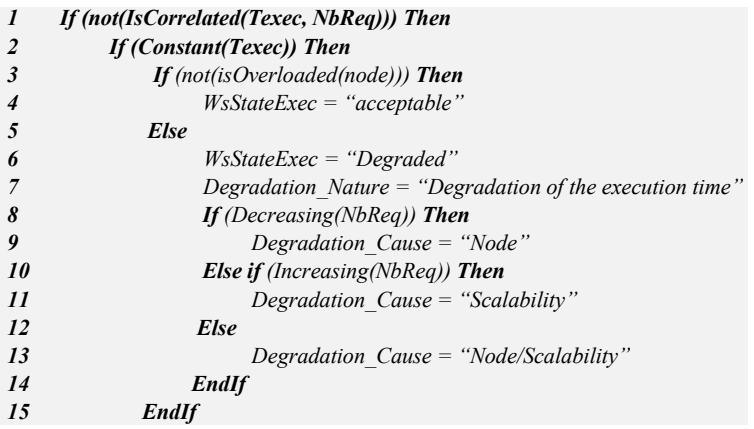

Fig. 7. Execution time analysis sub-algorithm - Part 2.3: The execution time is constant during the training period 


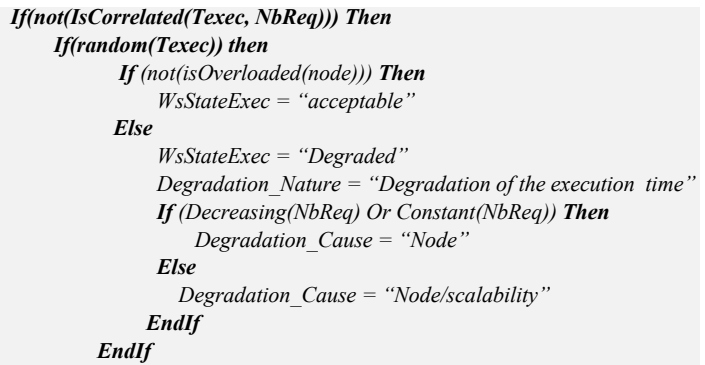

Fig. 8. Execution time analysis sub-algorithm - Part 2.4: The execution time is random during the training period

it deduces that the degradation comes from the hosting node (Fig. 7 line 9). Otherwise, if the simultaneous request number is increasing (Fig. [7, line 10), our sub-algorithm deduces that the QoS degradation is related to a scalability issue (Fig. 7, line 11). Else, (i.e., NbReq(t) is random), it concludes that the cause of the QoS degradation is related to a scalability issue and/or a hosting node overload (Fig. 7) line 13).

In Fig. 8, this sub-algorithm handles the case when Texec(t) is random and not correlated with $\mathrm{NbReq}(t)$. In order to evaluate the Web service state, it checks the hosting node load. If it is not overloaded (Fig. 88 line 3), our subalgorithm concludes that the state of the concerned Web service is acceptable (Fig. 8, line 4). In the opposite case (Fig. 8 line 5), our sub-algorithm deduces that a degradation of the execution time has occurred (Fig. 8 lines 6 and 7). In order to locate the degradation cause, it checks the variation of the simultaneous request number. If it is decreasing or constant (Fig. 8: line 8), it deduces that the hosting node is the degradation cause (Fig. 8 line 9). Otherwise (Fig. 8 line 10), we conclude that the degradation is related to a scalability issue and/or to a hosting node overload (Fig. 8: line 11). In the following, we present carried out experiments illustrating our approach.

\section{Illustration}

To validate our approach, we carried out two kinds of experiments. In the first one, we compare the manually generated QoS reference values with the QoS reference values automatically generated within our approach. We note that these reference values are so close. In the second experiment, we show that the analysis using the automatically generated QoS reference values is achieved rigorously. In fact our analysis approach detects QoS degradation and allows an accurate identification of its nature and its cause.

The cooperative reviewing process starts by searching a suitable conference for authors. So, they send requests to the ConfSearch Web service looking for appropriate conferences (topics, publisher, etc.). In this paper, we focus on the 
performance analysis of the ConfSearch Web service. The experiment platform is composed of three nodes. The first contains Intel Pentium Dual Core T2310 as CPU with 1,46 GHZ as frequency and operates under Windows XP. The two other nodes operate under Ubuntu Linux and have each other an Intel Pentium Dual Core as CPU with $3 \mathrm{GHZ}$ as frequency. The application is composed of three main actors. The analyzer is deployed under the first node and implements our algorithm. This node contains also the monitoring database. The second actor is the ConfSearch Web service which is deployed under the second node. The Web service client represents the third actor, which is deployed under the third node. We use Apache Tomcat5.5 as Web server, Axis1.4 as SOAP engine, Java1.5 as programming language and MySQL5 as database management system.

\subsection{Validation of the Automatically Generated QoS Reference Values}

This experiment consists in comparing the QoS reference values, which are automatically generated by our algorithm, with the manually generated QoS reference values. It is composed of two phases. In the first one, we manually establish the QoS reference values of the ConfSearch Web service while running this Web service in optimal conditions. So, we run respectively 2-24, 32, 34 and 42 requests. For each request, we monitor QoS parameters, extract its values and measure network and node loads using specific tools (our monitoring middleware [2, IPERF and a specific checking_node_load service). This first phase is carried out 10 times. The graphs Texec_manual_ref (baby blue line) of the Fig. 9 and Tcom_manual_ref (orange line) of the Fig.10]represent their average. The second phase corresponds to the training period. It consists in applying our approach in order to automatically generate the QoS reference values. During this phase, we have injected some transient violations in order to illustrate the capacity of our approach to eliminate the degraded QoS values from the generated reference values. We run respectively 2-24, 32, 34 and 42 requests. For each request, we monitor QoS parameters and measure node and network loads. For the execution time, we injected a delay on the ConfSearch Web service (while running several processes in the hosting node) in the $5^{\text {th }}, 9^{\text {th }}, 11^{\text {th }}, 14^{\text {th }}, 16^{\text {th }}$ and $20^{\text {th }}$ requests. For the communication time, we injected a network overload (while using the HPING4 tool) in the $5^{t h}, 9^{t h}, 18^{t h}, 20^{t h}, 21^{\text {st }}$ and $22^{\text {nd }}$ requests. This phase is also carried out 10 times. The graphs Texec(with transient violations) (blue line) of the Fig. 9 and Tcom(with transient violations) (red line) of the Fig. 10 show the experiment results that we conducted. At this phase end, our algorithm generates QoS reference values. They are shown in Fig. 9] (Texec_ref_algo) and 10 (Tcom_ref_algo). As presented in Fig. 9] our algorithm has correctly generated the execution time reference values (Tcom_ref_algo) (green line) which are very close to the manually generated one. In addition, Fig. 10 illustrates that the manual communication time reference values (Tcom_manual_ref) are very close to the communication time reference values generated by our algorithm

\footnotetext{
4 http://www.hping.org/
} 


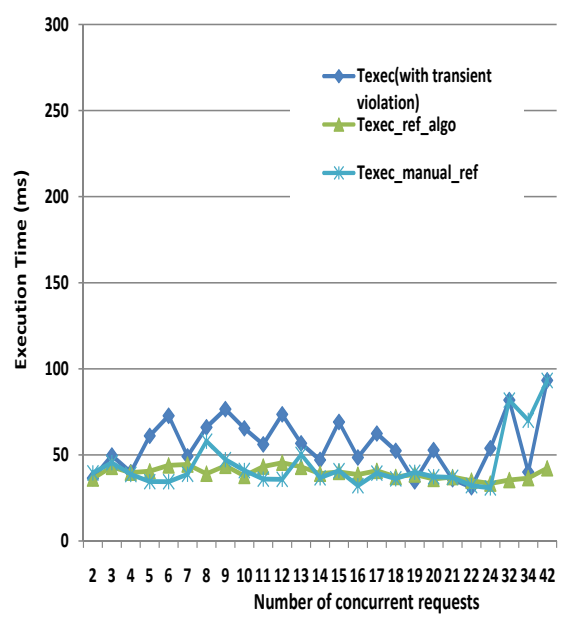

Fig. 9. Texec reference values

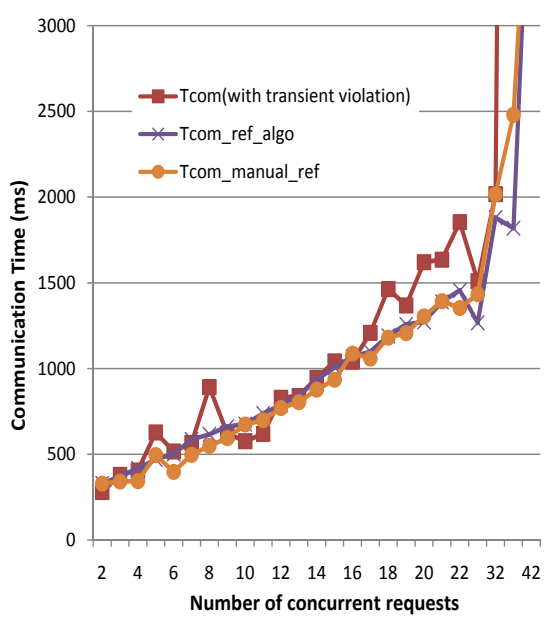

Fig. 10. Tcom reference values

(Tcom_ref_algo) (purple line). Fig. 9] and 10 illustrate that the degraded values (due to injected transient violations) are not selected for the generated QoS reference values. So, we conclude that the automation of QoS reference values generation gives an efficient result.

\section{$3.2 \quad$ Efficiency of Our Approach}

In order to illustrate the efficiency of our performance analysis approach, we injected three types of failures. This is detailed in the following.

-First failure: Network overload. This experiment consists in generating a flooding attack using the HPING tool while running our analyzer. We noticed that our algorithm detects a communication degradation and identifies its cause which is a network overload. This illustrates the efficiency of our approach.

-Second failure: Node overload. In this experiment, we generate a node failure and we follow the behavior of our analyzer. So, we run simultaneously a lot of processes on the ConfSearch Web service hosting node. The proposed algorithm detects an execution degradation and deduces its cause: node overload.

- Third failure: Scalability issue. In this experiment, we progressively increase the simultaneous request number to cause a scalability issue. We note that our analyzer detects the degradation and deduces its cause: a scalability issue.

This illustrates that our approach offers an accurate localization of the degradation cause. It checks the node load, the network load and the variation of the QoS parameters to allow this accurate identification of the degradation cause.

\section{Related Work}

Works dealing with the analysis of Web services may be classified in three categories. The first one is based on QoS parameters values comparison with 
pre-established thresholds [8]. The approach of Tian et al. 8] monitors QoS parameters and checks the pre-defined thresholds. Thus, a degradation is detected when monitored values exceed thresholds. This approach performs the detection of the degradation while using a pre-established QoS reference values and does not allow neither the characterization of its nature nor the localization of its cause. However, our approach does not use a pre-established reference values and allows degradation detection, its nature characterization and its cause localization.

The second category is based on the model basis diagnosis technique 94.577 . It models the acceptable behavior of the monitored Web service. Then, the failure is detected when a deviation from the pre-established reference mode $\sqrt[5]{5}$ detected. For instance, Yan et al. 9] use the BPEL specifications to model the normal behavior of the monitored Web service. When a deviation from the preestablished model is detected, a degradation is signaled. This solution enables the degradation detection and the faulty Web service localization. So, it does not allow an accurate localization of the degradation cause and uses a pre-established reference model. However, our approach does not use a pre-established reference model and allows an accurate localization of the degradation cause.

Finally, the third category [6] is based on the representation of the acceptable behavior of the monitored Web service by a set of policies. Degradation is assimilated to a deviation from the pre-established model. This work [6], contrary to our approach, relies on the use of pre-established QoS reference values and does not allow neither the characterization of the degradation nature, nor a precise localization of its cause.

We noticed that studied works proposed an analysis solution based on the use of a pre-established reference model (QoS reference values / reference model) and do not offer a precise localization of the degradation cause. All these works build manually their reference model before the real publication of the Web service which is used during the analysis phase. However, this reference model can not be the same for all contexts and it varies from a context to another. Especially, when we have to handle the analysis of mobile Web services. In this case, it is not judicious to use a single reference model for the analysis. In fact, the service mobility leads to a perpetual change of the hosting node which makes impossible the use of a pre-established reference model (The reference model is not the same for all nodes).

\section{Conclusion}

In this paper, we proposed an approach allowing the performance analysis at runtime. It automatically and dynamically generates the QoS reference values. These reference values enable the Web service state characterization. It detects performance degradation, identifies its nature and gives an accurate localization of its cause. Our approach regenerates the QoS reference values whenever the Web service changes its execution context. It differs from existing ones since it

${ }^{5}$ A reference model describes the Web service acceptable state under a specific runtime context. In our work, it corresponds to the QoS reference values. 
does not rely on the use of a pre-established QoS reference values and accurately identifies the degradation cause. Our analysis approach is merged within a selfhealing framework developed in previous work [2. Experiments are carried out to show the feasibility and the benefits of such approach. The QoS reference values that we generated automatically enables a rigorously performance analysis at runtime. In this work, we only deal with execution time and communication time as QoS parameters. Other QoS parameters (such as throughput, response time, availability and reliability) are out of the scope of this paper. In addition, during the training period, we do not know about the Web service state. We have to wait until the end of this period in order to check the Web service state.

As perspectives, we plan to extend our approach to include more QoS parameters while studying more Web service parameters. Also, we aim at experimenting our approach in a large scale environment like Grid'5000.

\section{References}

1. Kephart, J.O., Chess, D.M.: The vision of autonomic computing. Computer 36(1), 41-50 (2003)

2. Ben-Halima, R., Drira, K., Jmaiel, M.: A qos-oriented reconfigurable middleware for self-healing web services. In: ICWS 2008: Proceedings of the 2008 IEEE International Conference on Web Services, Beijing, China, pp. 104-111. IEEE Computer Society, Los Alamitos (2008)

3. Ben-Halima, R., Fki, E., Jmaiel, M., Drira, K.: Experiments results and large scale measurement data for web services performance assessment. In: Proceedings of the 14th IEEE Symposium on Computers and Communications (ISCC 2009), Sousse, Tunisia, pp. 83-88. IEEE Computer Society, Los Alamitos (July 2009)

4. Ardissono, L., Console, L., Goy, A., Petrone, G., Picardi, C., Segnan, M., Dupré, D.T.: Towards self-diagnosing web services. In: Proceedings of International Workshop on Self-Managed Systems and Services (SELFMAN 2005), Nice, France. IEEE Computer Society, Los Alamitos (2005)

5. Ardissono, L., Furnari, R., Goy, A., Petrone, G., Segnan, M.: Fault tolerant web service orchestration by means of diagnosis. In: Gruhn, V., Oquendo, F. (eds.) EWSA 2006. LNCS, vol. 4344, pp. 2-16. Springer, Heidelberg (2006)

6. Moga, A., Soos, J., Salomie, I., Dinsoreanu, M.: Adding self-healing behaviour to dynamic web service composition. In: Proceedings of the 5th WSEAS International Conference on Data Networks, Communication and Computers, Bucharest, Romania, pp. 206-211 (2006)

7. Pucel, X., Bocconi, S., Picardi, C., Daniele, Dupré, T., Travé-Massuyès, L.: Analyse de la diagnosticabilité des services Web. In: Workshop Artificial Intelligence and Web Intelligence (IAWI), Grenoble, France (2007)

8. Tian, W., Zulkernine, F., Zebedee, J., Powley, W., Martin, P.: Architecture for an autonomic web services environment. In: WSMDEIS, Miami, pp. 32-44 (2005)

9. Yan, Y., Cordier, M.O., Pencole, Y., Grastien, A.: Monitoring web service networks in a model-based approach. In: Third IEEE European Conference on Web Services, ECOWS (2005)

10. Ross, S.M.: Introduction to probability and statistics for engineers and scientists. Elsevier Academic Press, Amsterdam (2004) 